\title{
Asymmetric interference in concurrent time-to-contact estimation: Cousin or twin of the psychological refractory period effect?
}

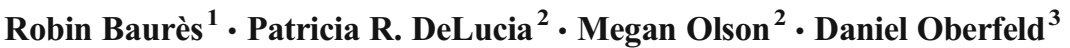

Published online: 28 November 2016

(C) The Psychonomic Society, Inc. 2016

\begin{abstract}
In a reaction time (RT) task requiring fast responses to two stimuli presented close in time, human observers show a delayed RT to the second stimulus. This phenomenon has been attributed to a psychological refractory period (PRP). A similar asymmetric interference is found when performing multiple concurrent visual time-to-contact (TTC) estimations for moving objects, despite important differences between the tasks. In the present study, we studied the properties of the asymmetric interference found in the TTC task and compared them to the classical PRP effect. In Experiment 1, we varied the time interval between the two objects' arrival times to determine the dependence of the PRP-like effect on the asynchrony between the two TTCs. In Experiment 2, we investigated whether the physical or the perceived arrival order determined the asymmetric interference. Our results confirmed the existence of asymmetric interference in the multiple TTC estimation task, but also indicated important differences from the traditional PRP effect observed in the RT paradigm. The origins of these differences are discussed, as well as the practical implications.
\end{abstract}

Keywords Time-to-contact estimation $\cdot$ Psychological refractory period $\cdot$ Dual-task interference, Prediction motion

Robin Baurès

robin.baures@cnrs.fr

CerCo, Université de Toulouse, CNRS, UPS, Toulouse, France

2 Texas Tech University, Lubbock, TX, USA

3 Experimental Psychology, Johannes Gutenberg-Universität Mainz, Mainz, Germany
It is often observed that the simultaneous performance of two tasks is less effective than the performance of a single task. This has been reported in both laboratory experiments (e.g., Maquestiaux, Laguë-Beauvais, Ruthruff, \& Bherer, 2008) and real-world tasks such as driving (e.g., Strayer, Drews, \& Johnston, 2003), in which distraction by a second task can have hazardous consequences (e.g., Klauer et al., 2014). Such effects have been attributed in part to a psychological refractory period (PRP; see, e.g., Pashler, 1994). The PRP effect consists of a delayed response to a second stimulus that follows a first stimulus after a brief time interval, analogous to the refractory period of neurons after action potential generation.

In a typical PRP experiment, two stimuli are presented successively with a variable temporal delay between them (the stimulus onset asynchrony, or SOA), and participants must respond to each stimulus as quickly as possible. This is termed the reaction time (RT) paradigm. For short SOAs (less than $100 \mathrm{~ms}$ ), the RT to the second stimulus is several hundred milliseconds longer than the RT to the same stimulus when it is presented alone. In contrast, the RT to the first stimulus is unaffected by the presence of the second stimulus. However, the increased RT to the second stimulus does not occur when the SOA is increased to several hundred milliseconds.

Many models have been proposed to explain the PRP effect (the central bottleneck model-e.g., Pashler, 1994; the capacity-sharing model - e.g., Tombu \& Jolicœur, 2003; and the strategic response-deferment model-Meyer \& Kieras, 1997a, 1997b). The first hypothesis is termed the central bottleneck model (e.g., Lien, Ruthruff, \& Johnston, 2006; Maquestiaux et al., 2008; Pashler, 1994). This model states that tasks are divided into three distinct processing stages: a precentral stage (e.g., stimulus identification), a central stage (e.g., response selection), and a postcentral stage (e.g., response execution). The precentral and postcentral stages of one task are assumed to operate in parallel with any stages 
of the other task. In contrast, processing in the central stage must be completed for one task before proceeding to the next. That is, the central stage of the second task cannot start until the central stage of the first task is completed, thus delaying the response to the second stimulus. This model predicts a delayed response (i.e., the RT) to the second stimulus at short SOAs only, because at long SOAs the processing in the central stage for the first task is already completed when the second stimulus is presented. The model also predicts that the response to the first object will remain unchanged if a second task is presented, because the tasks are processed in a "first come, first served" manner. For a diagram of the model, refer to Fig. 2 of Pashler (1994, p. 224).

The second hypothesis, the central capacity-sharing model (e.g., Navon \& Miller, 2002; Tombu \& Jolicœur, 2003, 2005), divides the task into the same three processing stages as the central bottleneck model, and all stages can be performed in parallel with any other stage of a secondary task. However, this model postulates that the central stage is capacity-limited, and therefore that the resources have to be split among the two central stages if they are performed at the same time- that is, at short SOAs. Consequently, both central stages can be conducted at the same time, but the reduction in the available resources slows down each central stage. Specifically, the first task can use the full capacity of the central task until Task 2 gains access to some of the central capacity. After some shared-capacity processing, Task 2 will then have full access to the central stage as soon as the processing of Task 1 is completed. Hence, both the RTs to both the first stimulus and the second stimulus should be increased at short SOAs, but less so at longer SOAs.

Finally, the strategic response-deferment model (Meyer \& Kieras, 1997a, 1997b) postulates that under specific conditions, it should be possible to achieve both tasks without interference. However, when these conditions are not met, a strategic bottleneck is implemented at some task stage in order to efficiently process both tasks. Hence, depending on the position of the bottleneck, the RT to the first and/or the second stimulus may be increased. This model does not make predictions distinct from those of the other two models, and we will not consider it further. This model can account for essentially the same effects (i.e., an increase in RT for the second stimulus) as the central bottleneck model (e.g., Tombu \& Jolicœur, 2003). In any case, it is not our goal to distinguish among the models, but rather to compare the PRP effect in the traditional RT task with a similar effect using a different task.

The PRP effect and its origins have been studied extensively since Telford's (1931) seminal work. To our knowledge, however, all of these studies have used an RT paradigm. Consequently, it is not known whether the PRP is specific to the RT paradigm, or is a more general process that occurs in other tasks. Recently, results compatible with a PRP process were found using a time-to-contact (TTC) estimation task
(Baurès, Oberfeld, \& Hecht, 2010, 2011). In these studies, participants performed two concurrent prediction motion (PM) tasks (Schiff \& Detwiler, 1979). They observed the motions of two objects that disappeared behind an occluder, and pressed a button when each object would reach a target location. The TTC estimate of the first-arriving object (the leading object) did not differ from the TTC estimate of the same object when it was presented alone. However, the TTC estimate of the later-arriving object (the trailing object) was longer than the TTC estimate of the same object when it was presented alone. Moreover, the delay in the TTC estimate of the trailing object was relatively greater when the difference between the two objects' arrival times at the finish line was relatively small. This pattern of results seems to reproduce the PRP pattern obtained in the RT paradigm, but it is not known whether they reflect a common underlying mechanism. To keep these phenomena distinct, we will use the term PRP effect to describe the results in the traditional RT paradigm, and use the term asymmetrical interference to describe the results in the PM paradigm.

In a PM task, participants traditionally respond at the exact time that a moving object appears to reach them or another location in the environment. According to ecological theories of TTC perception (Gibson, 1979), an object's TTC is directly specified by a property of the optic array, and it is not necessary for observers to rely on cognitive processes to estimate the TTC. For an object approaching the eye, the object's optical expansion pattern contains the "expansion tau," which is the ratio of the object's instantaneous optical size to its optical expansion rate (Hoyle, 1957; Lee, 1976). For an object moving laterally toward a target object, the decreasing optical gap between the moving object and the target contains the "gap tau" (Bootsma \& Oudejans, 1993). Such a theory does not consider possible limits in the use of this optical information from multiple objects that are judged concurrently (e.g., DeLucia \& Novak, 1997). According to Gibson's (1979) ecological approach to perception, the putatively limited pool of attentional resources (see, e.g., Carrasco, 2011) is not involved in TTC perception. Rather, "education of attention" occurs (e.g., Huet et al., 2011), in which observers learn to rely on more reliable information with practice. Thus, ecological theories predict that observers can estimate as many TTCs as there are moving objects, without accuracy being affected by the number of objects; asymmetric interference should not occur. However, experimental results contradict this prediction: Asymmetric interference was found when observers estimated the absolute TTC of two moving objects (Baurès et al., 2010, 2011).

It is important to recognize that the similarity between the PRP effect and asymmetrical interference was observed despite important differences in the two paradigms. In the RT paradigm, participants respond as soon as possible when each stimulus is presented. In the PM task, on the other hand, participants 
time their response to occur at the exact time that the moving object is anticipated to reach the target. This is an important difference, because the PM task is an anticipatory task, involving a predictive (anticipatory) response, rather than a simple RT. The PRP effect and the corresponding asymmetrical interference therefore seem to occur when two tasks have to be performed at the same time, irrespective of the nature of the task.

Despite the similarity, further investigation will be necessary before we can conclude that the PRP effect observed with the traditional RT paradigm and the asymmetrical inference observed when observers estimate the TTCs of multiple objects using the PM task are indeed the same phenomenon. In particular, two questions remain to be determined: the influence of the TTC occurrence asynchrony (TOA) on TTC estimation, and the influence of the perceived versus the physical arrival order of the stimuli.

First, in the traditional PRP paradigm, the presence of the first stimulus dramatically increases the RT to the second stimulus at short SOAs, and the RT to the second stimulus slowly returns to the RT measured for the first stimulus as the SOA increases. The dependency of the RT on the SOA can be approximated by a negatively accelerated decreasing function, with a slope approaching -1 at short SOAs (see Pashler, 1994, p. 222, Fig. 1). This implies that, for these short SOAs, every 1-ms increase in SOA leads to a 1-ms decrease in the RT to the second stimulus. In the PM task, the equivalent of the SOA is the difference between the two objects' actual TTCs, which we refer to as the TOA. So far, the shape of the TTC overestimation as a function of TOA remains unknown. In particular, it is not known for how long the first object influences the TTC estimation of the second. In addition, the magnitude of the influence at each TOA is not known. This was determined in Experiment 1 of the present study.

Second, an important feature of many traditional PRP studies is that humans are quite correct in judging the temporal order of two stimuli as soon as the temporal separation is above 100 ms (e.g., Hendrich, Strobach, Buss, Müller, \& Schubert, 2012; van Eijk, Kohlrausch, Juola, \& van de Par, 2008). Given the large range of SOAs (e.g., from 0 to $1,000 \mathrm{~ms}$ ) used in the RT paradigm, it is likely that observers' perceptions of the temporal order of the two stimuli matched the true order. In the PM task, however, it is not unusual for a proportion of the trials to reflect an incorrect perception of the order of the two objects' arrivals, even at much longer TOAs (e.g., $6 \%$ of the trials in Baurès et al., 2010, in which the minimal TOA was $500 \mathrm{~ms}$; these trials were excluded from the analysis). Thus, the PM task (but not the RT task) allows us to investigate whether asymmetrical interference is based on the physical or perceived arrival order. The PM task is ideal for this objective because it allows for the manipulation of participants' TTC estimates, such that the perceived and physical arrival orders differ. Specifically, TTC estimation depends not only on the actual TTC signaled by accurate visual cues like $\tau$, but also on heuristic cues (e.g., the size-arrival effect; DeLucia, 1991) and cognitive knowledge of an object's shape (López-Moliner, Brenner, \& Smeets, 2007), size (Hosking \& Crassini, 2011; López-Moliner, Field, \& Wann, 2007), velocity or occultation (Tijtgat, Bennett, Savelsbergh, De Clercq, \& Lenoir, 2010, 2011), and a trajectory (Hosking \& Crassini, 2010) or gravity effect (i.e., downward-moving objects are expected to accelerate, hence shortening their TTC estimations; Baurès \& Hecht, 2011; Zago et al., 2004). The goal of Experiment 2 of the present study is to investigate the role of perceived versus physical arrival order. We capitalized on the known effect of cognitive knowledge (i.e., gravity) on TTC estimates to induce a reversal in the perceived arrival order, and determine whether the asymmetric interference would then be observed for the first rather than the second stimulus.

\section{Experiment 1: Exploring the time course of asymmetric interference as a function of the TOA}

The PRP model states that the processing of the second task in the central stage is lengthened (capacity-sharing model) or postponed (central bottleneck model) until the processing of the first task in the central stage is completed. Accordingly, the PRP effect decreases as the SOA increases, which reduces the temporal difference between the end of the first task's processing and the start of the second task's processing. Although it was shown that the bottleneck in the multiple TTC estimation task originates from the sensory-processing stage (registration of the TTC-relevant optical variables) rather than the motor execution stage (Baurès et al., 2011), the largest TOA presented in this case was $1 \mathrm{~s}$. Thus, neither the duration of the bottleneck nor its magnitude at each TOA was determined. In Experiment 1 we presented a wider range of TOAs, to determine when the TTC estimation of the second object was no longer affected by the TTC estimation of the first object.

\section{Method}

Participants Sixteen students at Université Toulouse 3-Paul Sabatier (seven women, nine men) between 19 and 24 years of age $(M=21.56, S D=1.41)$ participated after giving informed consent. All of the participants had normal or corrected-tonormal vision and were healthy and without any known oculomotor abnormalities. The participants were naïve with respect to the purpose of the experiment, which was conducted in accordance with the Declaration of Helsinki.

Apparatus and experimental procedure This experiment was conducted with Cogent Graphics, developed by John Romaya at the Laboratory of Neurobiology at the Wellcome Department of Imaging Neuroscience. Stimuli were presented using a Hewlett Packard computer equipped with a $3.4-\mathrm{GHz}$ 
Intel i5 processor and a 27 -in. screen. The screen resolution was $1,024 \times 768$ pixels (horizontal by vertical). The monitor refresh rate and display update rate were $60 \mathrm{~Hz}$.

Participants sat on a chair and viewed the computer display from approximately $0.55 \mathrm{~m}$. The screen center was positioned midway between the two eyes. In the first condition (hereafter termed the one-object condition), participants estimated the TTC of a circular black object (diameter of $1 \mathrm{~cm}, 1$ degree of visual angle) that moved rightward at a constant speed in the frontoparallel plane against a white background. During its motion, the object passed behind an invisible rectangle ("occluder") that obscured its trajectory (cf., e.g., Baurès et al., 2010, Fig. 3, p. 365, who used visible occluders). Participants were asked to press the "B" key on a French keyboard at the instant the object would have collided with a vertically oriented black arrival line that was $0.3 \mathrm{~cm}$ wide and $15 \mathrm{~cm}$ long. The dimensions of the occluder varied according to the object's TTC.

Participants pressed the spacebar to start each trial. After a delay of 1,500 ms, the object appeared in a variable position at the left edge of the screen and moved toward the arrival line at either 3 or $6 \mathrm{~cm} / \mathrm{s}$. After 1,000 ms of visible motion, the object continued to move behind the occluder, and it reached the arrival line after $1,000,1,500,2,000,2,500$, or 3,000 ms. Once occluded, the object did not reappear. Participants indicated when the object would have hit the arrival line, and feedback was not provided. Ten unique scenes were created by factorially combining the two velocities with the five TTCs. Each scene was replicated five times, for a total of 50 trials.

After completing the one-object condition, participants completed the two-object condition. In this case, two objects were positioned one above the other, separated by $2 \mathrm{~cm}$, and moved rightward toward the arrival line. Participants were not given fixation instructions to focus on one particular object, and they were free to move their eyes as desired. They also were not instructed to favor the accuracy of one object's TTC estimation over the other's. Participants pressed the " $y$ " key to indicate when the upper object would reach the arrival line, and the " $b$ " key to indicate when the lower object would reach the line. The use of two different buttons has advantages and pitfalls. First of all, it allows an unambiguous measure of which object is perceived as arriving at the finishing line. In contrast, using a single button would require the assumption that the perceived arrival order matched the physical arrival order (i.e., that the shortest TTC was perceived as the shortest). A disadvantage of using two buttons is that deciding between two answers may induce a delay in the second answer, which could be incorrectly attributed to asymmetric interference. The rationale for using two buttons rather than one is based on the results of Baurès et al. (2011): As we described earlier, they showed that the bottleneck in the multiple TTC estimation task originates from the sensory-processing stage (registration of the TTC-relevant optical variables) rather than the motor execution stage (Baurès et al., 2011). Thus, we chose to ask the participants to use two buttons to give their answers. Feedback was not provided. As in the one-object condition, the visible movement time was $1,000 \mathrm{~ms}$, and each object moved at either 3 or $6 \mathrm{~cm} / \mathrm{s}$, for a total of four velocity conditions.

To maintain the number of trials in the two-object condition at a manageable level, one object always had a TTC of 2,000 ms. This served as the reference object: The TTC estimation of only this object was compared to the TTC estimation of the same object in the one-object condition. The TTC of the other object (the second object) was equal to $\mathrm{TTC}_{\text {ref }}$ TOA, where $\mathrm{TTC}_{\text {ref }}=2,000 \mathrm{~ms}$ was the TTC of the reference object, and the TOA was set to a value of $-500,+50,+100$, $+150,+250,+500,+750,+1,000,+1,250$, or $+1,500 \mathrm{~ms}$. This resulted in TTC values for the second object of 2,500, 1,950, 1,900, 1,850, 1,750, 1,500, 1,250, 1,000, 750, and $500 \mathrm{~ms}$, respectively. When the TOA was negative, the second object arrived after the reference object; when the TOA was positive, the second object arrived before the reference object. ${ }^{1}$

The combination of the reference object's two velocities and one TTC value resulted in two different trajectories. The combination of the second object's two velocities and ten TOAs resulted in 20 different trajectories. The sizes of the occluders were varied such that the TTC remained constant $(2,000 \mathrm{~ms})$ for the reference object, which resulted in the required TTC for the second object. Combining the single TTC of the reference object with the different TTCs of the second object resulted in 40 unique two-object scenes, each presented five times, for a total of 200 two-object trials. These 200 trials were split into five different blocks, with a break provided upon the participant's request, and the block order was randomly counterbalanced across participants. The position of the reference object (upper vs. lower) was balanced across the two-object condition.

\section{Results}

One-object condition We computed the constant error (CE) by subtracting the actual TTC from the estimated TTC on each

\footnotetext{
${ }^{1}$ This represents an important difference between the present study and our prior studies. In Baurès et al. $(2010,2011)$, the temporal separation between the two objects' arrivals was termed the $\Delta \mathrm{TTC}$, computed as the reference object's TTC minus the second object's TTC. A negative value indicated that the reference object arrived first, whereas a positive value indicated that the reference object arrived second. This was opposite to the terminology of the PRP literature, in which the time difference between the two stimuli is termed the stimulus onset asynchrony (SOA), and a 0 -ms value is defined as the occurrence of the reference stimulus. A negative value thus indicates that the second stimulus appeared first, and a positive value indicates that the second stimulus appeared second. Because the present article has a strong focus on asymmetric interference in the context of TTC estimation, as compared to the original PRP effect, we adopted the PRP terminology and used TOA instead of $\Delta$ TTC. Any comparison with the results of Baurès et al. (2010, 2011), should be made by inverting the meanings of positive and negative $\triangle \mathrm{TTC}$ : A positive $\Delta$ TTC corresponds to a negative TOA, and conversely, a negative $\Delta$ TTC corresponds to a positive TOA.
} 
trial, and analyzed CE with a 2 (Velocity: 3 or $6 \mathrm{~cm} / \mathrm{s}$ ) $\times 5$ (TTC: 1,000, 1,500, 2,000, 2,500, or 3,000 ms) repeated measures ANOVA. The Huynh-Feldt correction for the degrees of freedom was used where applicable (Huynh \& Feldt, 1976), and the value of $\tilde{\varepsilon}$ is reported. Post-hoc comparisons among all levels of TTC were conducted using nonpooled error terms (i.e., by computing separate paired-samples $t$ tests; Keselman, 1994) and Hochberg's (1988) sequentially acceptive step-up Bonferroni procedure, with an alpha level of .05.

As is shown in Fig. 1, we found an effect of TTC, $F(4,60)$ $=5.40, p<.001, \tilde{\varepsilon}=.47, \eta_{\mathrm{p}}{ }^{2}=.26$. On average, the TTC estimates were longer than the veridical values, but this overestimation decreased with increases in the actual TTC. Such a pattern is frequently found in PM tasks (e.g., Oberfeld, Hecht, \& Landwehr, 2011). The results also showed a significant influence of velocity, $F(1,15)=421.58, p<.001, \eta_{\mathrm{p}}{ }^{2}=.97$; the CE was larger for the slower velocity. TTC and velocity did not interact, $F(4,60)=0.62, p=.65$.

Two-object condition To analyze the influence of introducing a second object on TTC estimation, we focused our analysis on the TTC estimations of the reference object (which always had a TTC of 2,000 ms). For each velocity of the reference object, we subtracted the mean CE (computed from the five repetitions) observed in the one-object condition, for that velocity and the reference TTC value of 2,000 ms, from the mean CE for the reference object in the two-object condition. This was done for each participant and trajectory of the second object. For example, imagine that the first participant's mean CE was $100 \mathrm{~ms}$ in the oneobject condition when the object's TTC was 2,000 ms and its velocity was $3 \mathrm{~cm} / \mathrm{s}$. This same participant's mean $\mathrm{CE}$ was $-50 \mathrm{~ms}$ in the two-object condition when the

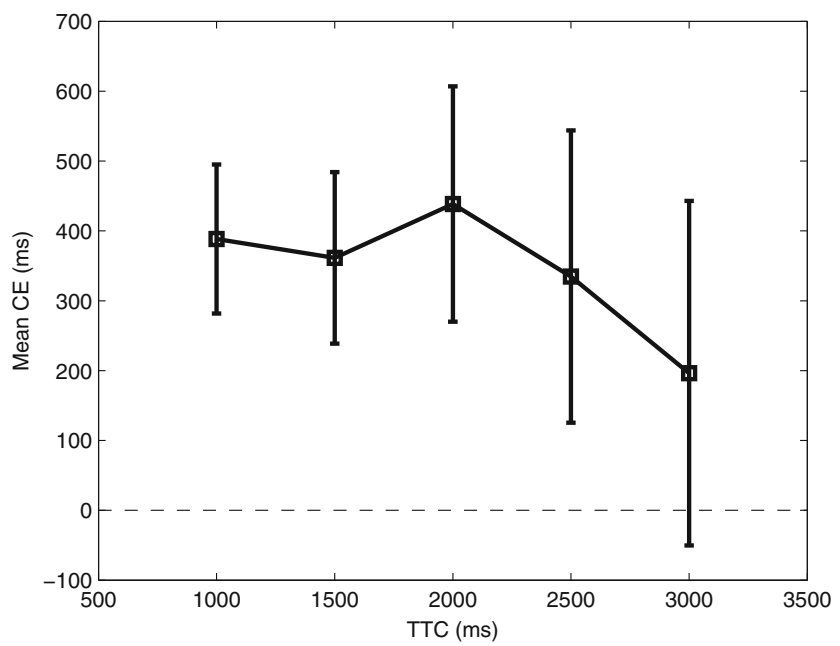

Fig. 1 Mean constant errors (CEs; estimated minus actual time to contact [TTC]) as a function of TTC in the one-object condition of Experiment 1. Error bars indicate the $95 \%$ confidence interval; therefore, error bars not covering 0 indicate that the mean value is significantly different from $0(p$ $<.05)$ reference object's TTC was 2,000 ms and its velocity was $3 \mathrm{~cm} / \mathrm{s}$, whereas the second object's TTC was $1,500 \mathrm{~ms}$ and its velocity was $6 \mathrm{~cm} / \mathrm{s}$. In this case, the change in $\mathrm{CE}$ due to the presence of the second object was $\triangle \mathrm{CE}=-50 \mathrm{~ms}-100 \mathrm{~ms}=-150 \mathrm{~ms}$. Notably, $\triangle \mathrm{CE}$ does not reflect the precision of the participant's TTC estimate, but rather the shift in the TTC estimate when the TTCs of two objects are judged, rather than just one. A positive value of $\triangle \mathrm{CE}$ indicates that the addition of the second object resulted in an increase in the $\mathrm{CE}$ relative to the one-object condition (i.e., a relative overestimation of the TTC in the two-object condition). Conversely, a negative value of $\triangle \mathrm{CE}$ indicates that the second object resulted in a decrease in the $\mathrm{CE}$ relative to the one-object condition (a relative underestimation).

We analyzed $\triangle \mathrm{CE}$ with a 2 (Reference-Object Velocity) $\times$ 2 (Second-Object Velocity) $\times 10$ (TOA) repeated measures ANOVA. The results indicated a significant effect of TOA, $F(9,135)=14.18, p<.001, \tilde{\varepsilon}=.45, \eta_{\mathrm{p}}{ }^{2}=.49$. As is shown in Fig. 2, $\triangle \mathrm{CE}$ was very small when the TOA was negative, had a sizeable positive value for small positive TOAs, and gradually decreased as the TOA increased to values greater than $1,000 \mathrm{~ms}$. Moreover, the values of $\triangle \mathrm{CE}$ for the comparable TOAs are consistent with those reported in Baurès et al. (2010). The post-hoc analysis is presented in Table 1 .

For each TOA, we conducted two-tailed, one-sample $t$ tests to compare the value of $\triangle \mathrm{CE}$ (averaged across velocity conditions) to $0 \mathrm{~ms}$. A $\triangle \mathrm{CE}$ close to 0 would indicate that the TTC estimation of the reference object did not differ significantly between the one-object and two-object conditions, and thus that the estimate of the reference object was not influenced by the second object. The mean values of $\triangle \mathrm{CE}$ and the lower and upper bounds of the $95 \%$ confidence intervals (CIs) are presented in Table 2. The inclusion of 0 in the 95\% CI indicates a nonsignificant difference. As can be seen in Table 2, the second object had a significant influence on the TTC estimation of the first object only when the TOA was between 50 and $750 \mathrm{~ms}$. In addition, the mean $\triangle \mathrm{CE}$ generally decreased as the TOA increased, as the asymmetric interference would predict.

We observed a main effect of reference-object velocity, $F(1,15)=95.29, p<.001, \eta_{\mathrm{p}}{ }^{2}=.86$ : The mean $\Delta \mathrm{CE}$ was greater for $6 \mathrm{~cm} / \mathrm{s}$ than for $3 \mathrm{~cm} / \mathrm{s}$. The second-object velocities showed a significant but opposite pattern, $F(1,15)=$ $21.81, p<.001, \eta_{\mathrm{p}}{ }^{2}=.59$, in which the mean $\Delta \mathrm{CE}$ was greater for $3 \mathrm{~cm} / \mathrm{s}$. There was also a significant interaction between the reference and second-object velocities, $F(1,15)=13.33, p$ $=.002, \eta_{\mathrm{p}}{ }^{2}=.47$, in which the influence of the referenceobject velocity was more pronounced when the second object's velocity was 6 rather than $3 \mathrm{~cm} / \mathrm{s}$. We also found a three-way interaction among TOA, reference-object velocity, and second-object velocity $[F(9,135)=12.23, p<.001, \tilde{\varepsilon}=$ $\left..82, \eta_{\mathrm{p}}{ }^{2}=.45\right]$, for which we have no explanation. 


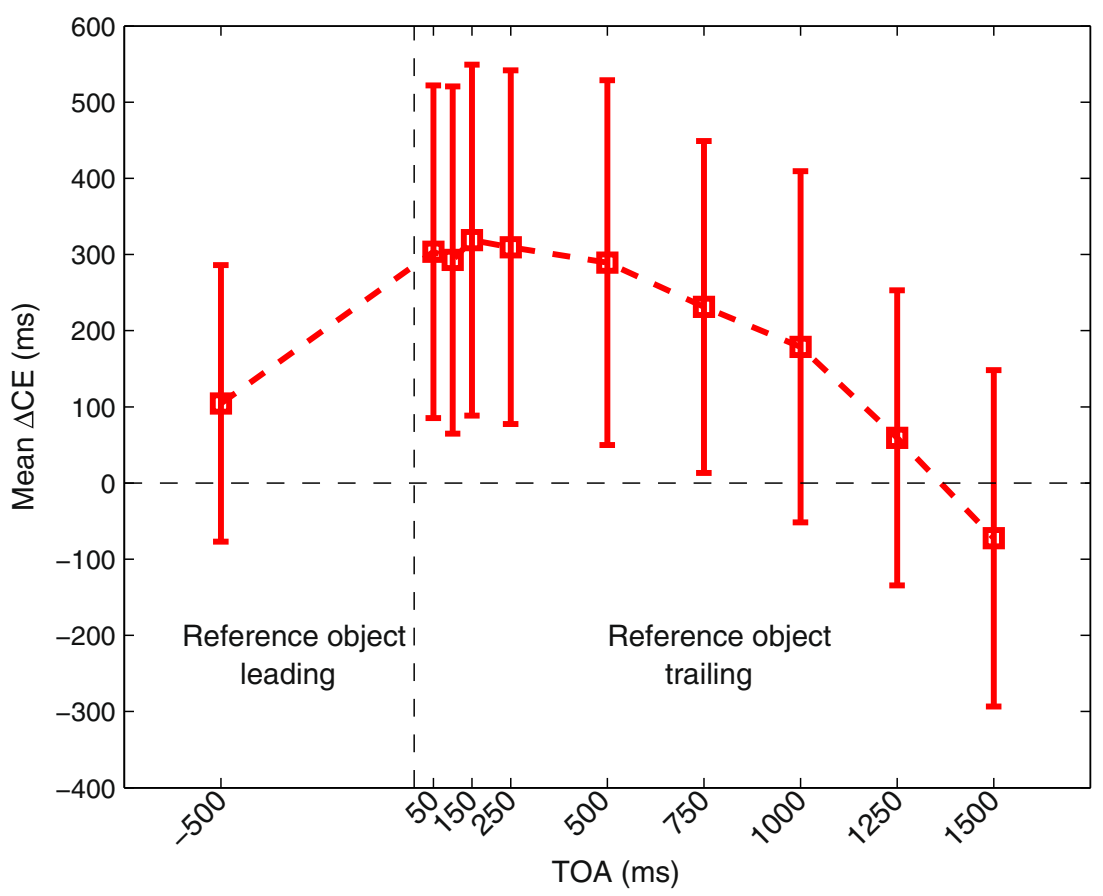

Fig. 2 Mean changes in constant error $(\triangle \mathrm{CEs})$ for the reference object (TTC $=2,000 \mathrm{~ms}$ ), as a function of TTC occurrence asynchrony (TOA), in the two-object condition of Experiment 1. Error bars represent the 95\%

\section{Discussion of Experiment 1}

The results of Experiment 1 confirmed the existence of an asymmetric interference process when participants estimate two TTCs at the same time. TTC estimates were significantly longer when the TOA was between 50 and $750 \mathrm{~ms}$, but were close to the TTC estimates in the one-object condition when the TOA was 1,250 or $1,500 \mathrm{~ms}$. The effect of the second object decreased as TOA increased, and on the basis of our data, the asymmetric interference due to the first object's arrival seems to vanish if the two TTCs are separated by more than $1,000 \mathrm{~ms}$. In

Table 1 Post-hoc analysis of the differences in $\triangle \mathrm{CE}$ between all pairs of the TTC occurrence asynchrony (TOA) presented in Experiment 1

\begin{tabular}{lllllllllll}
\hline TOA $(\mathrm{ms})$ & -500 & 50 & 100 & 150 & 250 & 500 & 750 & 1,000 & 1,250 & 1,500 \\
\hline-500 & & & & & & & & & & \\
50 & $*$ & & & & & & & & & \\
100 & $*$ & & & & & & & & & \\
150 & $*$ & & & & & & & & & \\
250 & $*$ & & & & & & & & \\
500 & $*$ & & & & & & & & \\
750 & & & & & & & & & \\
1,000 & & & & $*$ & $*$ & & & & \\
1,250 & & $*$ & $*$ & $*$ & $*$ & & $*$ & & & \\
1,500 & & $*$ & $*$ & $*$ & $*$ & $*$ & $*$ & $*$ & &
\end{tabular}

An asterisk indicates significant differences between the pairs of TOAs, identified with the Hochberg (1988) procedure confidence intervals. $\triangle \mathrm{CE}$ represents the difference in constant errors between the two-object condition and the one-object condition

addition, we found no influence of the second-arriving object's TTC estimation on the first-arriving object's TTC estimation (i.e., there was no significantly positive $\triangle \mathrm{CE}$ at negative TOAs). Our results appear consistent with the central bottleneck model (see, e.g., Pashler, 1994) rather than with the central capacity-sharing model (e.g., Navon \& Miller, 2002), which predicts increased TTC estimations for both objects. Alternatively, participants may have allocated all of their attention to the first-arriving object rather than sharing it between the two objects. In this case, the results are consistent with the

Table 2 Mean $\triangle \mathrm{CEs}$ and $95 \%$ confidence intervals (CIs) as a function of TOA

\begin{tabular}{llll}
\hline TOA (ms) & $\begin{array}{l}\text { Mean } \Delta \text { CE } \\
(\mathrm{ms})\end{array}$ & $\begin{array}{l}\text { Lower Bound } \\
\text { of the 95\% CI }\end{array}$ & $\begin{array}{l}\text { Upper Bound } \\
\text { of the 95\% CI }\end{array}$ \\
\hline-500 & 104.51 & -77.15 & 286.18 \\
50 & $303.59^{*}$ & 85.36 & 521.83 \\
100 & $292.82^{*}$ & 64.83 & 520.82 \\
150 & $318.81^{*}$ & 88.40 & 549.23 \\
250 & $309.54^{*}$ & 77.44 & 541.63 \\
500 & $289.31^{*}$ & 50.00 & 528.63 \\
750 & $231.08^{*}$ & 13.17 & 449.00 \\
1,000 & 178.82 & -51.67 & 409.31 \\
1,250 & 59.36 & -134.38 & 253.11 \\
1,500 & -72.54 & -293.38 & 148.29 \\
\hline
\end{tabular}

An asterisk indicates that the mean $\triangle \mathrm{CE}$ is significantly different from $0 \mathrm{~ms}$ - that is, $0 \mathrm{~ms}$ is not included in the $95 \% \mathrm{CI}$ 
capacity-sharing model, which makes the same predictions as the central bottleneck model. The present study was not designed to distinguish between these models.

The results do not completely match those from traditional PRP studies. In the latter, the decrease in RTs that occurs as SOA increases typically shows a large decrease of RT for the shorter SOA (i.e., a slope of -1 for the PRP effect at SOAs shorter than 200-300 ms), followed by a slow asymptotic return to the RT to the first stimulus at the longer SOAs (e.g., Pashler, 1994, p. 222, Fig. 1). Our present results show a very different pattern, as can be seen in Fig. 2. For TOAs between 50 and $250 \mathrm{~ms}, \triangle \mathrm{CE}$ remained high and approximately constant. Most of the decrease in $\triangle \mathrm{CE}$ occurred for the longer TOA values. This pattern is opposite to that observed in traditional PRP experiments. Although an effect of the SOA or TOA on the judgments of the second stimulus occurred in both the RT and PM paradigms, the patterns of the effect were different.

\section{Experiment 2: Role of perceived versus physical arrival order in the asymmetric interference}

In Experiment 1, and in prior studies of the asymmetric interference in the multiple TTC estimation task (Baurès et al., 2010, 2011), the pattern of results was described on the basis of the actual arrival order: The TTC of the first-arriving object was estimated correctly relative to a one-object condition, whereas the TTC of the second-arriving object was overestimated relative to the results in a one-object condition (particularly at short TOAs). This type of analysis and description implicitly assumes that the perceived arrival order is identical to the physical arrival order. However, the object with the objectively earlier TTC is the first object that requires an action only if the perceived arrival order does match the objective order. It is reasonable to expect that if the perceived versus the physical arrival order could be manipulated, the pattern of effects would change: If the physically first-arriving object appeared to arrive second, the overestimation of TTC would occur in the objectively first-arriving object rather than the objectively second-arriving object. The data from Baurès et al. (2010) are consistent with such an effect. In their experiment, the presence of a second object requiring a TTC estimate resulted in a relative overestimation of the TTC (as compared to a one-object condition) for the trailing object, but not for the leading object. This effect was even more pronounced, however, when their analyses excluded trials in which the TTC estimates indicated that the perceived did not match the objective arrival order (see Baurès et al., 2010, Fig. 7, p. 368). Reversing the apparent arrival times of the first and second objects would be difficult to achieve in the traditional RT paradigm, but it can be achieved with the PM task by manipulating the object's trajectory to induce the known effect of knowledge on TTC estimation.
Specifically, an object that moves downward along a vertical trajectory (i.e., parallel to the force of gravity) results in a shorter TTC estimate than does the same object moving along a horizontal trajectory, presumably because the vertical path triggers an internal model of gravity, imputing acceleration to the object (e.g., Zago et al., 2004). Such data are compatible with the idea that observers are estimating the TTC of an accelerating object rather than of one moving at a constant velocity, independently of the actual kinematics of the object (e.g., McIntyre, Zago, Berthoz, \& Lacquaniti, 2001; Zago et al., 2004); this occurs especially when the occlusion time is long (e.g., Baurès \& Hecht, 2011; Bosco, Delle Monache, \& Lacquaniti, 2012). The results typically show a 50 -ms (e.g., Bosco et al., 2012; McIntyre et al., 2001) to 150-ms (e.g., Le Séac'h, Senot, \& McIntyre, 2010; Senot, Zago, Lacquaniti, \& McIntyre, 2005) difference in TTC estimations between the conditions in which gravity is and is not induced. In Experiment 2, we included objects that moved from the top to the bottom of the display, as well as objects that moved from the left to the right of the display. We expected that we could reverse the perceived arrival order of the two moving objects, and consequently manipulate the object that was influenced by the asymmetric interference.

In the first condition, hereafter referred to as the vertical screen orientation, two different object trajectories were presented. As in Experiment 1, the objects moved on a computer screen in the frontoparallel plane of the participant. One trajectory was from the left to the right edge of the display, and the other trajectory was from the top to the bottom edge of the display, aligned with gravity. The range of TOAs was varied to ensure that the magnitude of the "gravity effects" known from previous studies could reverse the perceived arrival order. In the case of short TOAs leading to a reversal in the perceived arrival order, we expected that the asymmetric interference would affect the judgment of the physically firstarriving object. A control condition was included in which the screen's orientation was not upright, but was lying flat on a table. Participants stood up and looked at the screen from above at the exact same visual stimuli as in the first condition. In this condition-hereafter termed the horizontal screen orientation - the object moved from the top to the bottom edge of the display, and thus did not have a trajectory aligned with gravity (Fig. 3). In this case, the internal model of gravity should be ineffective (e.g., Baurès \& Hecht, 2011; Le Séac'h et al., 2010; Senot et al., 2005), and the perceived arrival order should perfectly match the physical arrival order. Accordingly, the asymmetric interference should only affect the TTC estimation of the second-arriving object.

\section{Method}

Participants Twelve students at Université Toulouse 3-Paul Sabatier (six women, six men) between 20 and 30 years of age 


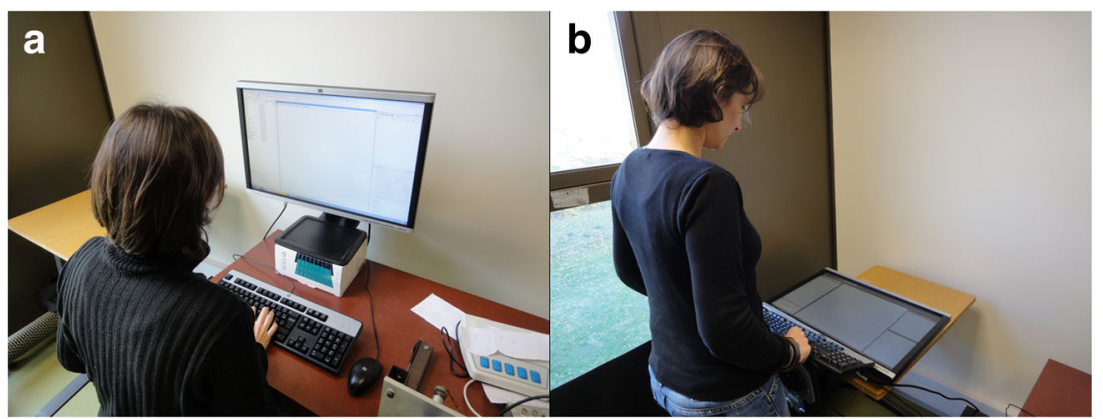

Fig. 3 Illustration of the two screen conditions of Experiment 2. (A) Vertical screen condition, in which a movement trajectory from top to bottom was aligned with gravity. (B) Horizontal screen condition, in which the movement trajectory from top to bottom was not aligned with gravity

$(M=22.58, S D=0.84)$ participated after giving informed consent. All participants had normal or corrected-to-normal vision and were healthy and without any known oculomotor abnormalities. Participants were naïve with respect to the purpose of the experiment, which was conducted in accordance with the Declaration of Helsinki.

Apparatus and experimental procedure For the experiment, we used the same devices as in the first experiment. Because most of the protocols of Experiments 1 and 2 were identical, we will only discuss the differences. In the one-object condition, the object moved at either 2 or $4 \mathrm{~cm} / \mathrm{s}$ (instead of 3 and $6 \mathrm{~cm} / \mathrm{s}$ ). After a visible movement time of $1,000 \mathrm{~ms}$, the object passed behind the occluder and continued its movement to reach the arrival line after $1,500,2,000$, or 2,500 ms (instead of 1,000 to 3,000 ms, in 500-ms steps, in Exp. 1). The two velocities and three TTCs were combined to create six unique scenes, each presented five times. These trials were presented in two blocks, representing different screen orientations, for a total of 60 trials. Depending on the screen orientation condition, participants faced either a screen oriented vertically (Fig. 3A), or a screen oriented horizontally (Fig. 3B) on a table with the image facing upward (toward the ceiling) and viewed from above by the participants. Such a manipulation would induce an expectation of gravity when the stimulus moved from the upper to the lower edge of the display in the vertical screen orientation, but not when it moved in the horizontal screen orientation (Amorim et al., 2015).

In the two-object condition, both objects were presented simultaneously. Their trajectories were perpendicular, with one moving on the screen from left to right, and the other moving from top to bottom (Fig. 4). Participants were not given fixation instructions to focus on one particular object, and they were free to move their eyes as desired. They also were not instructed to favor the accuracy of one object's TTC estimation over the other's. The two paths intersected at the arrival point. The reference object always moved from left to right and had a TTC of 2,000 ms. The second object moved from top to bottom, and its TTC was equal to $\mathrm{TTC}_{\mathrm{ref}}-\mathrm{TOA}$, where TOA was set to values of $-500,-250,-150,-50,+50$, $+150,+250,+500,+750$, or $+1,000 \mathrm{~ms}$. This resulted in TTC values for the second object of 2,500, 2,250, 2,150, 2,050, $1,950,1,850,1,750,1,500,1,250$, and 1,000 ms, respectively. As in Experiment 1, when the TOA was negative, the second object arrived after the reference object; when the TOA was positive the second object arrived before the reference object. Combining the single TTC of the reference object with the different TTCs of the second objects resulted in 40 unique two-object scenes, each presented five times, for a total of 200 two-object trials. In addition, these trials were presented in both the vertical screen orientation and the horizontal screen orientation conditions, with order of gravity (vertical vs. horizontal screen orientation conditions) counterbalanced across the participants. Participants thus performed 400 trials in the two-object condition. The two screen orientation conditions were completed on separate days. For a given screen orientation, the participants always began with the one-object trials, followed by the two-object trials.

\section{Results}

One-object condition We analyzed the CEs with a 2 (Screen Orientation Condition) $\times 3($ TTC) $\times 2$ (Velocity) repeated measures ANOVA. The results showed an influence of velocity, $F(1,11)=680.32, p<.001, \eta_{\mathrm{p}}{ }^{2}=.98$, with smaller errors for the faster velocity. None of the remaining effects or interactions was significant. Importantly, the CEs were consistent with those obtained in Experiment 1.

Two-object condition The $\triangle \mathrm{CE}$ was computed as in Experiment 1 and analyzed with a 2 (Screen Orientation Condition) $\times 2$ (Reference-Object Velocity) $\times 2$ (SecondObject Velocity) $\times 10($ TOA) repeated measures ANOVA. The effect of screen orientation condition was not significant, $F(1,11)=0.06, p=.82$. We observed an effect of TOA, $F(9$, 99) $=110.97, p<.001, \tilde{\varepsilon}=.41, \eta_{\mathrm{p}}{ }^{2}=.91$, showing the expected increase in $\triangle \mathrm{CE}$ for positive TOAs, followed by a gradual decrease as the TOA increased, consistent with Experiment 1. 


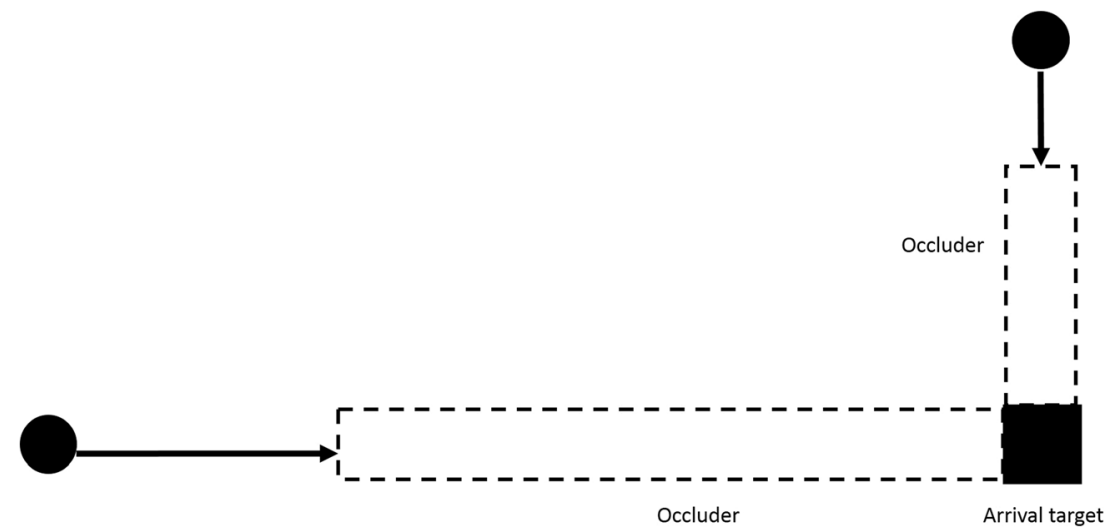

Fig. 4 Representation of the two-object condition of Experiment 2. Two balls were moving, one from left to right and the other from up to down, and were occluded by two occluders (dashed rectangles). Participants had

Importantly, there was a significant TOA $\times$ Screen Orientation interaction, $F(9,99)=28.24, p<.001, \tilde{\varepsilon}=.90, \eta_{\mathrm{p}}{ }^{2}=.72$. As is shown in Fig. 5 , the $\triangle \mathrm{CE}$ changed as a function of TOA, with approximately the same trends in the vertical and horizontal screen orientation conditions. However, the rise of the curve began slightly sooner in the vertical than in the horizontal screen orientation condition, at TOA $=-50 \mathrm{~ms}$ in the former condition and TOA $=+50 \mathrm{~ms}$ in the latter condition.

Post-hoc pairwise comparisons using the Hochberg (1988) procedure showed that the $\triangle \mathrm{CEs}$ differed significantly between the vertical and horizontal screen orientation conditions only when the TOA was $-50 \mathrm{~ms}$. In this condition, the effect of the screen orientation was very strong, with a mean difference of $759.17 \mathrm{~ms}$ (95\% CI: [510.17, 1,008.18] ms). As in to press a button for each ball when they were thought to collide with an arrival square (in black). In the one-object condition, only the horizontally moving ball was present

Experiment 1, we conducted $t$ tests to compare the mean $\triangle \mathrm{CEs}$ to $0 \mathrm{~ms}$. The mean values of $\triangle \mathrm{CE}$ and lower and upper bounds of the $95 \%$ CI are presented in Table 3 . In the vertical screen orientation condition, the $\triangle \mathrm{CEs}$ differed from 0 for TOAs between -50 and $+50 \mathrm{~ms}$, indicating that asymmetric interference was triggered even for the negative TOA. In contrast, in the horizontal screen orientation condition, $\triangle \mathrm{CEs}$ differed from 0 for positive TOAs between +50 and $+250 \mathrm{~ms}$, and not for $-50 \mathrm{~ms}$. These results suggest that in the vertical screen orientation condition, the expected perceived acceleration of the object due to the knowledge of gravity distorted the perceived arrival order when the TOA was $-50 \mathrm{~ms}$. We interpreted this result as indicating that the object that physically arrived first was perceived as arriving
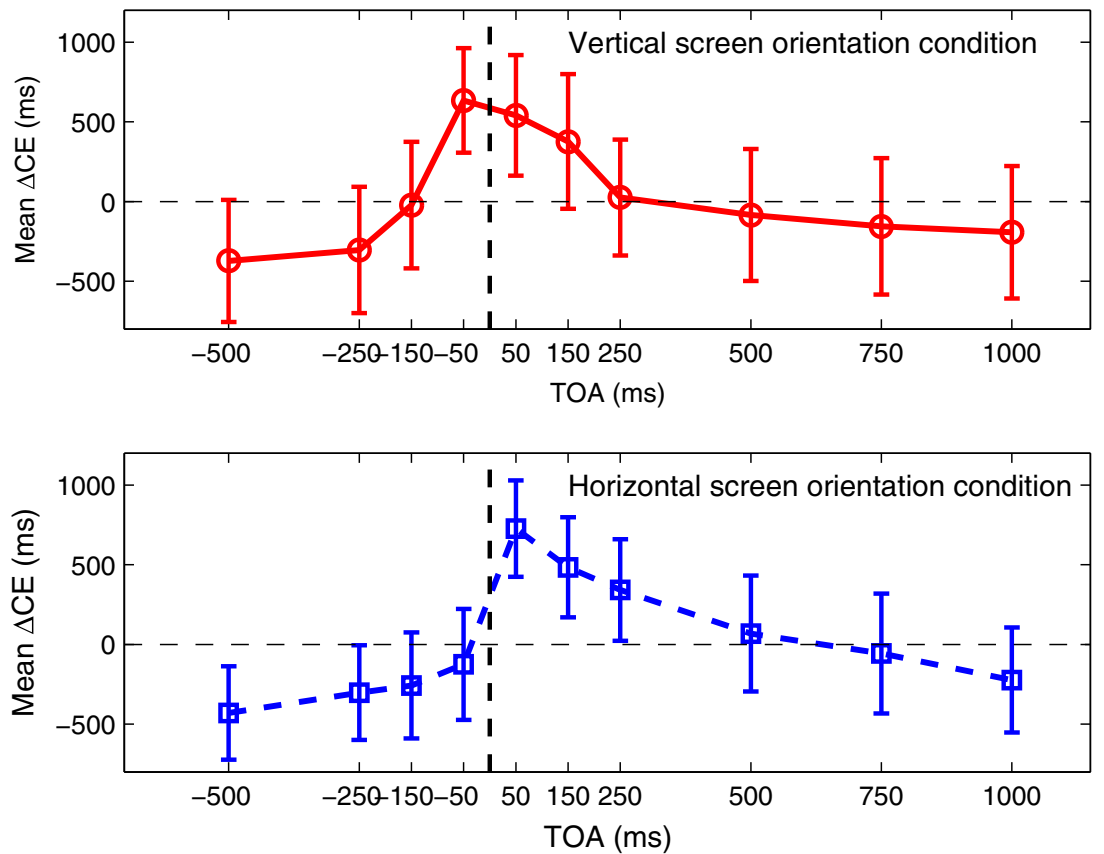

Fig. 5 Mean $\triangle \mathrm{CEs}$ for the reference object moving from left to right, as a function of TOA (negative values = reference object leading), in the vertical, gravity-aligned (upper panel) and horizontal (lower panel) screen orientation conditions of Experiment 2. The error bars represent the $95 \%$ confidence intervals 
Table 3 Mean $\triangle \mathrm{CEs}$ and 95\% confidence intervals (CIs) as a function of TOA and screen orientation condition of the Experiment 2

\begin{tabular}{|c|c|c|c|c|c|c|}
\hline \multirow[b]{2}{*}{ TOA (ms) } & \multicolumn{3}{|c|}{ Vertical Screen Orientation Condition } & \multicolumn{3}{|c|}{ Horizontal Screen Orientation Condition } \\
\hline & Mean $\triangle \mathrm{CE}$ & $\begin{array}{l}\text { Lower Bound } \\
\text { of the } 95 \% \text { CI }\end{array}$ & $\begin{array}{l}\text { Upper Bound } \\
\text { of the } 95 \% \text { CI }\end{array}$ & Mean $\triangle \mathrm{CE}$ & $\begin{array}{l}\text { Lower Bound } \\
\text { of the } 95 \% \text { CI }\end{array}$ & $\begin{array}{l}\text { Upper Bound } \\
\text { of the } 95 \% \text { CI }\end{array}$ \\
\hline-500 & -372.54 & -755.05 & 9.98 & $-430.59^{*}$ & -723.58 & -137.61 \\
\hline-250 & -304.27 & -700.31 & 91.77 & $-302.93^{*}$ & -599.78 & -6.09 \\
\hline-150 & -22.53 & -419.24 & 374.18 & -257.71 & -590.32 & 74.89 \\
\hline-50 & $633.73^{*}$ & 306.06 & 961.39 & -125.45 & -473.67 & 222.77 \\
\hline 50 & $539.94^{*}$ & 161.77 & 918.11 & $726.35^{*}$ & 423.27 & $1,029.43$ \\
\hline 150 & 376.45 & -46.39 & 799.29 & $483.34^{*}$ & 169.74 & 796.94 \\
\hline 250 & 25.22 & -338.31 & 388.74 & $341.15^{*}$ & 23.21 & 659.09 \\
\hline 50 & -84.17 & -497.90 & 329.57 & 68.16 & -296.10 & 432.43 \\
\hline 750 & -155.49 & -583.67 & 272.69 & -57.28 & -432.59 & 318.04 \\
\hline 1,000 & -193.00 & -607.85 & 221.85 & -223.64 & -552.99 & 105.71 \\
\hline
\end{tabular}

An asterisk indicates that the mean $\triangle \mathrm{CE}$ is significantly different from $0 \mathrm{~ms}$ - that is, $0 \mathrm{~ms}$ is not included in the $95 \% \mathrm{CI}$

second, resulting in an overestimation of TTC due to the asymmetric interference. The results are consistent with our expectation that the perceived rather than the physical arrival order was the basis of the effect. This assumption predicts a delayed TOA effect (e.g., an effect when the TOA is $150 \mathrm{~ms}$, but not yet when it is $50 \mathrm{~ms}$ ) if the reference object moves from top to bottom, which is a condition we did not include to limit the duration of the experiment.

The ANOVA also revealed an influence of reference-object velocity, $F(1,11)=328.32, p<.001, \eta_{\mathrm{p}}{ }^{2}=.97$, as well as of second-object velocity, $F(1,11)=50.52, p<.001, \eta_{\mathrm{p}}{ }^{2}=.82$. The pattern of results was similar to that observed in Experiment 1: The faster reference-object velocity and the slower second-object velocity resulted in a relatively greater $\triangle \mathrm{CE}$ than the slower reference-object velocity and the faster second-object velocity, respectively.

We found a significant two-way interaction, TOA $\times$ Reference-Object Velocity, $F(9,99)=3.11, p=.002, \tilde{\varepsilon}=$ $.78, \eta_{\mathrm{p}}{ }^{2}=.22$. There were also two three-way interactions: Screen Orientation Condition $\times$ TOA $\times$ Reference-Object Velocity, $F(9,99)=2.21, p=.03, \tilde{\varepsilon}=.91, \eta_{\mathrm{p}}{ }^{2}=.17$, and TOA $\times$ Reference-Object Velocity $\times$ Second-Object Velocity, $F(9,99)=6.09, p<.001, \tilde{\varepsilon}=.71, \eta_{\mathrm{p}}{ }^{2}=.36$. However, none of them exhibited interesting patterns of $\Delta$ CEs with respect to our hypotheses.

\section{Discussion of Experiment 2}

The PRP effect, or its asymmetric interference counterpart, consists of an increase in RT (in the traditional PRP paradigm) or an increase in TTC estimation (in the multiple TTC estimation paradigm) of the second-arriving object, whereas the response to the first-arriving object remains broadly constant. The order of stimulus onsets (RT paradigm) and the order of
TTCs (PM paradigm) seem to be crucial factors in the asymmetric interference. In Experiment 2, we manipulated the object's trajectory to putatively reverse the perceived arrival order, and found support for our hypothesis: When acceleration due to gravity was suggested by the second object's trajectory (i.e., moving down on a vertical display aligned with gravity), the asymmetric interference (i.e., the increase in $\triangle \mathrm{CE}$ ) occurred for a negative TOA - that is, when objectively the reference object arrived first. In contrast, when acceleration due to gravity was not implied by the second object's trajectory (i.e., moving from top to bottom of a display lying flat on a table), the same negative TOA did not result in an increase in $\Delta \mathrm{CE}$, compatible with the assumption that the perceived arrival order matched the physical order.

The results of Experiment 2 further allowed us to approximate the TTC underestimation due to the gravitational influence that putatively occurred to compensate for the occlusion period in the PM paradigm (Zhao \& Warren, 2015). The asymmetric interference that occurred for the negative TOA suggests that the object moving from top to bottom was perceived as arriving first, even when its TTC was $50 \mathrm{~ms}$ longer than that of the object moving from left to right. However, such a misperception did not seem to occur when the TTC of the object moving from top to bottom was $150 \mathrm{~ms}$ longer than that of the other object. This suggests that the gravitational influence shortens TTC estimation by a magnitude within this range of 50 to $150 \mathrm{~ms}$, a finding compatible with the usual values reported in experiments investigating the internal model of gravity (e.g., Lacquaniti \& Maioli, 1989; McIntyre et al., 2001; Senot et al., 2005; Zago et al., 2004; see Baurès, Benguigui, Amorim, \& Siegler, 2007, for a review). Figure 6 illustrates this: When the data points for the horizontal screen orientation are shifted to the left by $100 \mathrm{~ms}$, the $\Delta \mathrm{CE}$ values for the two screen orientations align quite nicely. 


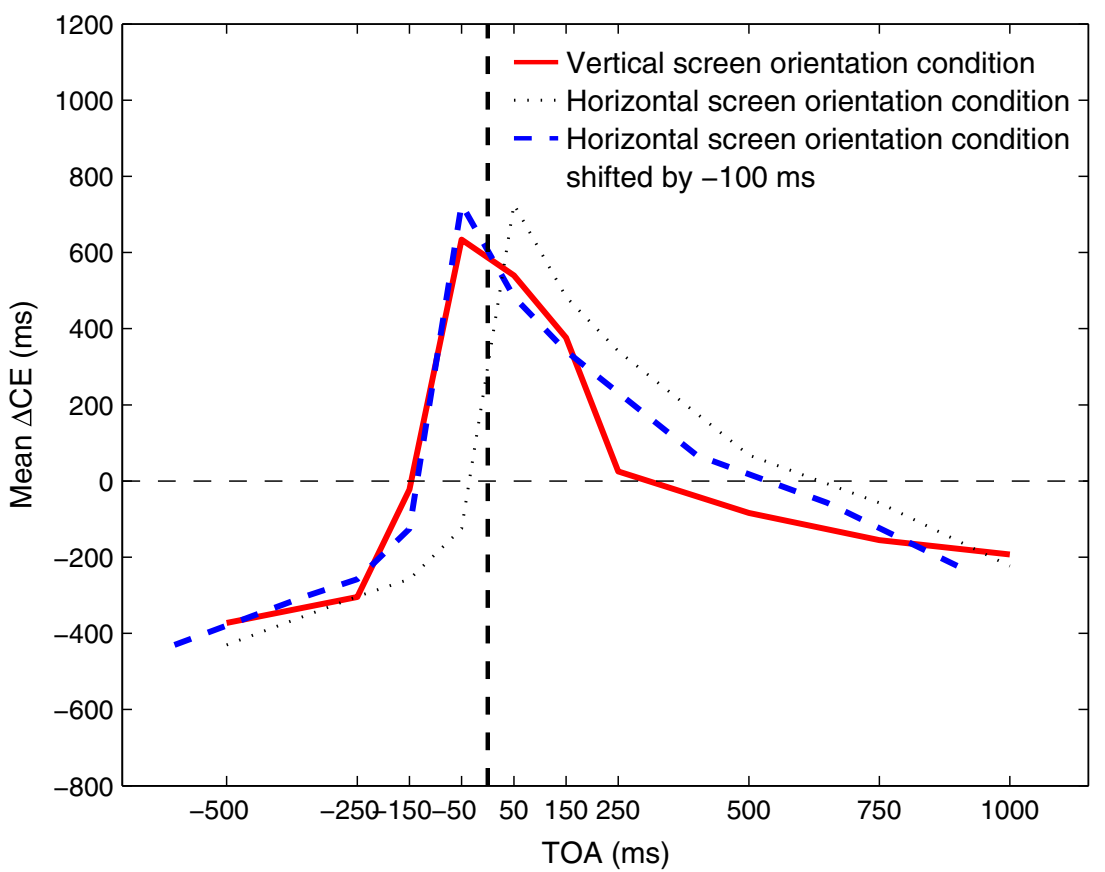

Fig. 6 Mean $\triangle$ CEs in the vertical (solid line) and horizontal (dashed line) screen orientation conditions, when the data points of the horizontal screen orientation condition (dotted line) are shifted to the left by $100 \mathrm{~ms}$

Difference between Experiments 1 and 2 Although the results of Experiments 1 and 2 demonstrated the asymmetric interference, they also exhibited important differences. In Experiment 1, the maximum amplitude of the $\triangle \mathrm{CE}$ due to asymmetric interference was $318.81 \mathrm{~ms}$ (95\% CI: [88.4, 549.23]); in Experiment 2, the maximum amplitude of $726.35 \mathrm{~ms}$ was much greater (95\% CI: [423.27, 1,029.43]). In contrast, the asymmetric interference endured for $750 \mathrm{~ms}$ in Experiment 1, but only for approximately $200 \mathrm{~ms}$ in Experiment 2. This suggests a trade-off between the asymmetric interference's duration and magnitude. It now becomes important to determine the parameters that influence this trade-off. A potential candidate is task difficulty, in terms of the capacity to visually access the two TTCs. In Experiment 1, the two objects moved on parallel trajectories, separated by a small vertical distance that putatively permitted efficient perception of the TTCs of both objects. Indeed, it has recently been shown that observers can efficiently fixate on one object while accessing another object's TTC if the two objects are temporally or spatially close (Baurès, Bennett, \& Causer, 2015). In Experiment 2, the two objects were distant from each other, likely making it much more difficult for participants to share visual attention between the two objects while maintaining their gaze on one of the objects. The implication is that the participants in Experiment 1 extracted the TTCs from the two objects at the same time, whereas the participants in Experiment 2 had to switch their gaze from one object to the other and maintain the unseen object's TTC in memory. The latter strategy might be harder, and it could contribute to the observed trade-off between the duration and magnitude of the asymmetric interference. According to this hypothesis, as difficulty increases, the magnitude of the asymmetric interference increases, but its duration decreases. This hypothesis remains to be empirically assessed.

\section{General discussion}

In prior research, when participants estimated two TTCs at the same time with a PM task, there was a delayed estimation for the second-arriving object (as compared to the estimation for just one object), whereas the estimation for the first-arriving object remained unaffected (Baurès et al., 2010, 2011). Such asymmetric interference resembles a PRP effect, typically demonstrated with RT tasks in which participants react as quickly as possible to two stimuli. We examined the similarities and discrepancies between the effects observed in the two paradigms. Two properties of the asymmetric interference were tested: (1) At what value of the TTC occurrence asynchrony does the effect of the first-arriving object on the second-arriving object dissipate - that is, what is the asymmetric interference's duration? (2) Is the asymmetric interference a result of the perceived or the physical arrival order of the two objects?

Our results confirmed the existence of asymmetric interference in the multiple TTC estimation task, but also indicated important differences from the traditional PRP effect observed in the RT paradigm. First, we demonstrated a trade-off between the duration of the asymmetric interference and its magnitude, which has not been reported using RT tasks. Another 
difference lies in the relationship between TOA and $\triangle \mathrm{CE}$. In traditional PRP experiments, the slope of the function relating RT and SOA is approximately -1 at short SOAs, followed by a gradual decrease of the PRP effect for longer SOAs, a relationship that can be approximated by a $1 / x$ function. In our present findings, the slope of the relationship was instead approximated by a parabolic function: a large and approximately constant influence of TOA upon $\triangle \mathrm{CE}$ for the shorter TOAs, followed by a much smaller influence as the TOA increased. The approximately constant influence of TOA at its lower range is in firm opposition to the traditional PRP patterns found using the RT paradigm.

Second, our results suggest that the perceived arrival order of the two stimuli, and not the physical arrival order, is the basis of the asymmetric interference, which has not been anticipated by ecological theories of perception. Indeed, this observation is in fundamental contradiction with Gibson's (1979) approach to visual perception, according to which perception is veridical (e.g., Epstein \& Park, 1964) — that is, perfectly matches the visual scene. In this respect, using the optical cues from the scene should allow the perceived order to match the physical order, in contradiction with the present data. However, this finding is consistent with previous results showing that visual illusions may affect TTC estimation (DeLucia, Tresilian, \& Meyer, 2000). In a Sander parallelogram, the two diagonals were incorrectly perceived as having different lengths, and the TTC estimation of an object moving along the left (apparently longer) diagonal was larger than the TTC estimation made when the object moved along the right (apparently smaller) diagonal (for large occlusion times). Hence, our present results confirm that (mis)perception of the environment properties can influence TTC estimation. More generally, our results confirm the dramatic influence of the first TTC estimation upon a second TTC estimation, in apparent contradiction with the ecological theory. The latter does not consider the putatively limited pool of attentional resources that allows access to only one TTC at a given time.

Why should asymmetric interference increase the TTC estimation of the second-arriving object? In the RT paradigm in which the classical PRP effect is observed, observers are required to respond as quickly as possible to each of the two stimuli. Thus, if, as is proposed by the central bottleneck idea (Pashler, 1994), a processing stage is blocked by the processing of the first-presented stimulus, the processing of the second-presented stimulus can only start after completion of the processing of Stimulus 1, and this leads to a delayed response to Stimulus 2. In the PM paradigm, it is more difficult to explain the systematic overestimation of the TTC of the second-arriving object in the two-object condition. As was explained by Baurès et al. (2011), the PM task requires (1) sensory registration of the TTC-relevant optical variables, (2) the computation of an absolute TTC estimate based on the information about the objects' motion extracted during Stage
1, (3) preparation/timing of the motor response to coincide with the estimated TTC, and (4) initiation and execution of the buttonpress indicating the estimated TTC. If the capacity of Stage 1 or 2 is limited, so that the first-arriving object is processed preferentially, this might result in a less precise TTC estimate for the second-arriving object. However, it is difficult to explain why the "blocking" of Stage 1 or 2 should cause a systematic overestimation of the trailing object's TTC, rather than simply an increased variance of this TTC estimate. Concerning Stage 3, researchers debate whether the observers in a PM task use motion extrapolation in the sense of visual imagery, or whether they estimate the TTC at the moment the object disappears from the screen, and then use a timing mechanism to delay their response until the virtual arrival time (e.g., DeLucia \& Liddell, 1998; Tresilian, 1995). If a timing mechanism was involved, a systematic overestimation of the TTC of the second-arriving object would be plausible, because concurrent temporal and nontemporal tasks have been shown to result in longer productions of temporal intervals than in a single-interval timing condition (e.g., Brown, 1997; Champagne \& Fortin, 2008). In terms of pacemaker-accumulator models of time perception (Gibbon, Church, \& Meck, 1984; Zakay \& Block, 1997), if the observer focuses attention on the timing of the response for the first-arriving object in Stage 3, this could cause pulses from the internal clock/ pacemaker for the second-arriving object to be missed (Zakay \& Block, 1997). Thus, fewer "clock ticks" would reach the accumulator involved in the timing of the response to the second-arriving object, and as a consequence the threshold for producing the response would be reached later, resulting in an overestimation of the second-arriving object's TTC. However, it is not entirely clear whether timing processes play a role in PM tasks (DeLucia \& Liddell, 1998). Also, in a task in which two moving objects were presented but the object for which a TTC estimate had to be produced was cued either before the start of the trial or at occlusion onset, Baurès et al. (2011) found systematic overestimation of the TTC of the second-arriving object, although attention sharing in Stage 3 was not required, because only a single TTC estimate had to be produced. Thus, additional research will be needed to identify the cause of the overestimation of the second-arriving object's TTC in the two-object condition. Taken together, the conclusion from Experiments 1 and 2 is that the asymmetric interference observed in a PM task requiring two concurrent TTC estimates is a cousin rather than a twin of the classical PRP effect observed in the RT paradigm.

It is important to consider that eye movements could also play a role in the occurrence of asymmetrical interference. Indeed, it has been demonstrated that eye movements influence performance in PM tasks, as compared to a fixation condition (e.g., Bennett, Baurès, Hecht, \& Benguigui, 2010; Makin \& Chauhan, 2014; Makin \& Poliakoff, 2011). Although we did not monitor eye movements, and therefore 
are not able to confirm this hypothesis, it seems unlikely that the participants in the present study fixated the two objects throughout a given trial, because of the optical distance between them (both of the objects did not fall within the fovea). Two strategies could be expected: At the beginning of a trial, participants could use saccades to switch their fixation from one object to the other to determine which had the shortest TTC, and subsequently could maintain fixation on this firstarriving object only. It has been shown that the TTC discrimination threshold is affected by an object's eccentricity in the visual field (Regan \& Vincent, 1995); hence, the lack of foveal input on the second-arriving object could contribute to the observed asymmetrical interference. A second strategy is that participants could use saccades to switch their fixations from one object to the other throughout the entire trial, resulting in equivalent fixation times for the two objects. It has been demonstrated that during saccades, time perception is compressed (Morrone, Ross, \& Burr, 2005); thus, making saccades throughout the trial could impair TTC estimation. However, it is unclear why, under the latter hypothesis, the influence of saccades would lead to asymmetrical interference rather than affect both TTC estimations. A replication of our experiment while eye movements are monitored is warranted, to gain more knowledge about how eye movements contribute to the asymmetrical interference.

In conclusion, estimating multiple TTCs at the same time leads to an asymmetric interference of one object upon a second one: The first-arriving object's TTC estimation influenced the second-arriving object's, similar to the traditional PRP pattern. However, the dependence of this effect on the TTC asynchrony showed notable differences from the effect of the SOA on the PRP effect observed in a traditional RT paradigm. Finally, the asymmetric interference in the PM task depends on the objects' perceived, rather than their physical, arrival order.

\section{References}

Amorim, M.-A., Siegler, I. A., Baurès, R., \& Oliveira, A. M. (2015). The embodied dynamics of perceptual causality: A slippery slope? Frontiers in Psychology, 6, 483. doi:10.3389/fpsyg.2015.00483

Baurès, R., Benguigui, N., Amorim, M.-A., \& Siegler, I. A. (2007). Intercepting free falling objects: Better use Occam's razor than internalize Newton's law. Vision Research, 47, 2982-2991. doi:10.1016/j.visres.2007.07.024

Baurès, R., Bennett, S. J., \& Causer, J. (2015). Temporal estimation with two moving objects: Overt and covert pursuit. Experimental Brain Research, 233, 253-261. doi:10.1007/s00221-014-4110-y

Baurès, R., \& Hecht, H. (2011). The effect of body posture on longrange time-to-contact estimation. Perception, 40, 674-681. doi: $10.1068 / \mathrm{p} 6945$

Baurès, R., Oberfeld, D., \& Hecht, H. (2010). Judging the contact-times of multiple objects: Evidence for asymmetric interference. Acta Psychologica, 134, 363-371. doi:10.1016/j.actpsy.2010.03.009
Baurès, R., Oberfeld, D., \& Hecht, H. (2011). Temporal-range estimation of multiple objects: Evidence for an early bottleneck. Acta Psychologica, 137, 76-82. doi:10.1016/j.actpsy.2011.03.002

Bennett, S. J., Baurès, R., Hecht, H., \& Benguigui, N. (2010). Eye movements influence estimation of time-to-contact in prediction motion. Experimental Brain Research, 206, 399-407.

Bootsma, R. J., \& Oudejans, R. R. (1993). Visual information about timeto-collision between two objects. Journal of Experimental Psychology: Human Perception and Performance, 19, 1041-1052. doi:10.1037/0096-1523.19.5.1041

Bosco, G., Delle Monache, S., \& Lacquaniti, F. (2012). Catching what we can't see: Manual interception of occluded fly-ball trajectories. PLoS ONE, 7, e49381. doi:10.1371/journal.pone.0049381

Brown, S. W. (1997). Attentional resources in timing: Interference effects in concurrent temporal and nontemporal working memory tasks. Perception \& Psychophysics, 59, 1118-1140. doi:10.3758/BF03205526

Carrasco, M. (2011). Visual attention: The past 25 years. Vision Research, 51, 1484-1525. doi:10.1016/j.visres.2011.04.012

Champagne, J., \& Fortin, C. (2008). Attention sharing during timing: Modulation by processing demands of an expected stimulus. Perception \& Psychophysics, 70, 630-639. doi:10.3758/pp.70.4.630

DeLucia, P. R. (1991). Pictorial and motion-based information for depth perception. Journal of Experimental Psychology: Human Perception and Performance, 17, 738-748. doi:10.1037/00961523.17.3.738

DeLucia, P. R., \& Liddell, G. W. (1998). Cognitive motion extrapolation and cognitive clocking in prediction motion tasks. Journal of Experimental Psychology: Human Perception and Performance, 24, 901-914. doi:10.1037/0096-1523.24.3.901

DeLucia, P. R., \& Novak, J. B. (1997). Judgments of relative time-tocontact of more than two approaching objects: Toward a method. Perception \& Psychophysics, 59, 913-928. doi:10.3758/BF03205508

DeLucia, P. R., Tresilian, J. R., \& Meyer, L. E. (2000). Geometrical illusions can affect time-to-contact estimation and mimed prehension. Journal of Experimental Psychology: Human Perception and Performance, 26, 552-567. doi:10.1037/0096-1523.26.2.552

Epstein, W., \& Park, J. (1964). Examination of Gibson's psychological hypothesis. Psychological Bulletin, 62, 180-196.

Gibbon, J., Church, R. M., \& Meck, W. H. (1984). Scalar timing in memory. Annals of the New York Academy of Sciences, 423, 52-77.

Gibson, J. J. (1979). The ecological approach to visual perception. Boston, MA: Houghton Mifflin.

Hendrich, E., Strobach, T., Buss, M., Müller, H. J., \& Schubert, T. (2012). Temporal-order judgment of visual and auditory stimuli: Modulations in situations with and without stimulus discrimination. Frontiers in Integrative Neuroscience, 6, 63. doi:10.3389/fnint.2012.00063

Hochberg, Y. (1988). A sharper Bonferroni procedure for multiple tests of significance. Biometrika, 75, 800-802. doi:10.1093/biomet/75.4.800

Hosking, S. G., \& Crassini, B. (2010). The effects of familiar size and object trajectories on time-to-contact judgements. Experimental Brain Research, 203, 541-552. doi:10.1007/s00221-010-2258-7

Hosking, S. G., \& Crassini, B. (2011). The influence of optic expansion rates when judging the relative time to contact of familiar objects. Journal of Vision, 11(6), 20:1-13. doi:10.1167/11.6.20

Hoyle, F. (1957). The black cloud. London, UK: Heinemann.

Huet, M., Jacobs, D. M., Camachon, C., Missenard, O., Gray, R., \& Montagne, G. (2011). The education of attention as explanation of variability of practice effects: Learning the final approach phase in a flight simulator. Journal of Experimental Psychology: Human Perception and Performance, 37, 1841-1854. doi:10.1037/a0024386

Huynh, H., \& Feldt, L. S. (1976). Estimation of the Box correction for degrees of freedom from sample data in randomized block and splitplot designs. Journal of Educational and Behavioral Statistics, 1, 69-82. doi:10.3102/10769986001001069 
Keselman, H. J. (1994). Stepwise and simultaneous multiple comparison procedures of repeated measures' means. Journal of Educational and Behavioral Statistics, 19, 127-162. doi:10.3102/10769986019002127

Klauer, S. G., Guo, F., Simons-Morton, B. G., Ouimet, M. C., Lee, S. E., \& Dingus, T. A. (2014). Distracted driving and risk of road crashes among novice and experienced drivers. New England Journal of Medicine, 370, 54-59. doi:10.1056/NEJMsa1204142

Lacquaniti, F., \& Maioli, C. (1989). The role of preparation in tuning anticipatory and reflex responses during catching. Journal of Neuroscience, 9, 134-148.

Le Séac'h, A. B., Senot, P., \& McIntyre, J. (2010). Egocentric and allocentric reference frames for catching a falling object. Experimental Brain Research, 201, 653-662. doi:10.1007/s00221009-2081-1

Lee, D. N. (1976). A theory of visual control of braking based on information about time-to-collision. Perception, 5, 437-459. doi: 10.1068 /p050437

Lien, M.-C., Ruthruff, E., \& Johnston, J. C. (2006). Attentional limitations in doing two tasks at once: The search for exceptions. Current Directions in Psychological Science, 15, 89-93. doi:10.1111/j.09637214.2006.00413.x

López-Moliner, J., Brenner, E., \& Smeets, J. B. J. (2007). Effects of texture and shape on perceived time to passage: Knowing "what" influences judging "when.". Perception \& Psychophysics, 69, 887894. doi:10.3758/BF03193925

López-Moliner, J., Field, D. T., \& Wann, J. P. (2007). Interceptive timing: Prior knowledge matters. Journal of Vision, 7(13), 11:1-8. doi:10.1167/7.13.11

Makin, A. D. J., \& Chauhan, T. (2014). Memory-guided tracking through physical space and feature space. Journal of Vision, 14(13), 10. doi: $10.1167 / 14.13 .10$

Makin, A. D. J., \& Poliakoff, E. (2011). Do common systems control eye movements and motion extrapolation? Quarterly Journal of Experimental Psychology, 64, 1327-1343. doi:10.1080/17470218.2010.548562

Maquestiaux, F., Laguë-Beauvais, M., Ruthruff, E., \& Bherer, L. (2008). Bypassing the central bottleneck after single-task practice in the psychological refractory period paradigm: Evidence for task automatization and greedy resource recruitment. Memory \& Cognition, 36, 1262-1282. doi:10.3758/MC.36.7.1262

McIntyre, J., Zago, M., Berthoz, A., \& Lacquaniti, F. (2001). Does the brain model Newton's laws? Nature Neuroscience, 4, 693-694. doi: $10.1038 / 89477$

Meyer, D. E., \& Kieras, D. E. (1997a). A computational theory of executive cognitive processes and multiple-task performance: Part 1. Basic mechanisms. Psychological Review, 104, 3-65. doi:10.1037/0033-295X.104.1.3

Meyer, D. E., \& Kieras, D. E. (1997b). A computational theory of executive cognitive processes and multiple-task performance: Part 2. Accounts for psychological refractory-period phenomena. Psychological Review, 104, 749-791. doi:10.1037/0033-295 X.104.4.749

Morrone, M. C., Ross, J., \& Burr, D. C. (2005). Saccadic eye movements cause compression of time as well as space. Nature Neuroscience, 8 , 950-954.

Navon, D., \& Miller, J. (2002). Queuing or sharing? A critical evaluation of the single-bottleneck notion. Cognitive Psychology, 44, 193-251. doi:10.1006/cogp.2001.0767
Oberfeld, D., Hecht, H., \& Landwehr, K. (2011). Effects of task-irrelevant texture motion on time-to-contact judgments. Attention, Perception, \& Psychophysics, 73, 581-596. doi:10.3758/s13414-010-0040-3

Pashler, H. (1994). Dual-task interference in simple tasks: Data and theory. Psychological Bulletin, 116, 220-244. doi:10.1037/00332909.116.2.220

Regan, D., \& Vincent, A. (1995). Visual processing of looming and time to contact throughout the visual field. Vision Research, 35, 18451857.

Schiff, W., \& Detwiler, M. L. (1979). Information used in judging impending collision. Perception, 8, 647-658. doi:10.1068/p080647

Senot, P., Zago, M., Lacquaniti, F., \& McIntyre, J. (2005). Anticipating the effects of gravity when intercepting moving objects: Differentiating up and down based on nonvisual cues. Journal of Neurophysiology, 94, 4471-4480. doi:10.1152/jn.00527.2005

Strayer, D. L., Drews, F. A., \& Johnston, W. A. (2003). Cell phoneinduced failures of visual attention during simulated driving. Journal of Experimental Psychology: Applied, 9, 23-32. doi:10.1037/1076-898X.9.1.23

Telford, C. W. (1931). The refractory phase of voluntary and associative responses. Journal of Experimental Psychology, 14, 1-36. doi:10.1037/h0073262

Tijtgat, P., Bennett, S. J., Savelsbergh, G. J. P., De Clercq, D., \& Lenoir, M. (2010). Advance knowledge effects on kinematics of onehanded catching. Experimental Brain Research, 201, 875-884. doi:10.1007/s00221-009-2102-0

Tijtgat, P., Bennett, S. J., Savelsbergh, G. J., De Clercq, D., \& Lenoir, M. (2011). To know or not to know: influence of explicit advance knowledge of occlusion on interceptive actions. Experimental Brain Research, 214, 483-490. doi:10.1007/s00221-011-2846-1

Tombu, M., \& Jolicœur, P. (2003). A central capacity sharing model of dual-task performance. Journal of Experimental Psychology: Human Perception and Performance, 29, 3-18. doi:10.1037/00961523.29.1.3

Tombu, M., \& Jolicœur, P. (2005). Testing the prediction of the central capacity sharing model. Journal of Experimental Psychology: Human Perception and Performance, 31, 790802. doi:10.1037/0096-1523.31.4.790

Tresilian, J. R. (1995). Perceptual and cognitive processes in time-tocontact estimation: Analysis of prediction-motion and relative judgment tasks. Perception and Psychophysics, 57, 231-245. doi:10.3758/BF03206510

van Eijk, R. L. J., Kohlrausch, A., Juola, J. F., \& van de Par, S. (2008). Audiovisual synchrony and temporal order judgments: Effects of experimental method and stimulus type. Perception \& Psychophysics, 70, 955-968. doi:10.3758/PP.70.6.955

Zago, M., Bosco, G., Maffei, V., Iosa, M., Ivanenko, Y. P., \& Lacquaniti, F. (2004). Internal models of target motion: Expected dynamics overrides measured kinematics in timing manual interceptions. Journal of Neurophysiology, 91, 1620-1634. doi:10.1152/jn.00862.2003

Zakay, D., \& Block, R. A. (1997). Temporal cognition. Current Directions in Psychological Science, 6, 12-16. doi:10.1111/14678721.ep11512604

Zhao, H., \& Warren, W. H. (2015). On-line and model-based approaches to the visual control of action. Vision Research, 110(Pt. B), 190-202. doi:10.1016/j.visres.2014.10.008 